\title{
6 \\ DUFOUR AND SORET EFFECTS ON MHD FREE CONVECTIVE HEAT AND MASS TRANSFER FLOW PAST A VERTICAL POROUS FLAT PLATE EMBEDDED IN A POROUS MEDIUM
}

\author{
Md. Shariful Alam ${ }^{1}$ and Mohammad Mansur Rahman ${ }^{2}$ \\ ${ }^{1}$ Department of Mathematics, Dhaka University of Engineering and Technology (DUET), Gazipur-1700, \\ Bangladesh, Email: msalam631@yahoo.com \\ ${ }^{2}$ Department of Mathematics, University of Dhaka, Dhaka-1000, Bangladesh, Email: mansurdu@yahoo.com
}

\begin{abstract}
A two-dimensional steady MHD free convection and mass transfer flow past a semi-infinite vertical porous plate in a porous medium has been studied numerically including the Dufour and Soret effects. The resulting momentum, energy and concentration equations are then made similar by introducing the usual similarity transformations. These similar equations are then solved numerically by using the Nachtsheim-Swigert shooting method along with Runge-Kutta sixth order integration scheme. The numerical results are displayed graphically showing the effects of various parameters entering into the problem. Finally, the local values of the skin-friction coefficient $\left(C_{f}\right)$, Nusselt number (Nu) and Sherwood number (Sh) are also shown in tabular form.
\end{abstract}

Keywords: MHD, Free convection, Vertical plate, Steady flow, Porous medium, Dufour effect, Soret effect.

\begin{tabular}{llll}
\hline NOMENCLATURE: & & \\
$b$ & Empirical constant & $S c$ & Schmidt number \\
$B_{0}$ & Magnetic field intensity & $S h$ & Sherwood number \\
$\mathrm{C}$ & Concentration & $S r$ & Soret number \\
$c_{p}$ & Specific heat at constant pressure & $T$ & Temperature \\
$c_{s}$ & Concentration susceptibility & $T_{m}$ & Mean fluid temperature \\
$D a$ & Local Darcy number & $U_{0}$ & Uniform velocity \\
$D_{m}$ & Mass diffusivity & $u, v$ & Darcian velocities in the $x$ and $y$-direction \\
& & & respectively \\
$D u$ & Dufour number & $x, y$ & Cartesian coordinates along the plate and \\
& & & normal to it, respectively \\
$f_{w}$ & Dimensionless suction velocity & $\alpha$ & Thermal diffusivity \\
$F s_{I}$ & Local Forchheimer number & $\beta$ & Coefficient of thermal expansion \\
$g$ & Acceleration due to gravity & $\beta^{*}$ & Coefficient of concentration expansion \\
$G r$ & Local Grashof number & $\sigma$ & Electrical conductivity \\
$G m$ & Local modified Grashof number & $\rho$ & Density of the fluid \\
$K$ & Darcy permeability & $v$ & Kinematic viscosity \\
$k_{T}$ & Thermal diffusion ratio & $\theta$ & Dimensionless temperature \\
$M$ & Magnetic field parameter & $\phi$ & Dimensionless concentration \\
$N u$ & Nusselt number & $w$ & Condition at wall \\
$P r$ & Prandtl number & $\infty$ & Condition at infinity \\
$R e_{I}$ & Local Reynods number & &
\end{tabular}

\section{Introduction}

The study of Magnetohydrodynamic (MHD) flows have stimulated considerable interest due to its important applications in cosmic fluid dynamics, meteorology, solar physics and in the motion of Earth's core [Cramer \& Pai (1973)]. In a broader sense, MHD has applications in three different 
subject areas, such as astrophysical, geophysical and engineering problems. In light of these applications, steady MHD free convective flow past a heated vertical flat plate has been studied by many researchers such as Gupta (1961), Lykoudis (1962), and Nanda and Mohanty (1970). Raptis and Kafoussias (1982) studied free convection and mass transfer flow through a porous medium in the presence of transverse magnetic field, due to the importance of mass transfer and that of applied magnetic field in the study of star and planets. Recently Sattar et al. (2001) obtained similar solutions of a steady MHD free convection and mass transfer flow with viscous dissipation. They have used the perturbation method to solve the problem.

However, in all the above studies, Dufour and Soret effects were neglected, on the basis that they are of a smaller order of magnitude than the effects described by Fourier's and Fick's laws. There are, however, exceptions. The Soret effect, for instance, has been utilized for isotope separation and in mixture between gases and with very light molecular weight $\left(\mathrm{H}_{2}, \mathrm{He}\right)$, and for medium molecular weight $\left(\mathrm{H}_{2}\right.$, air) the Dufour effect was found to be of considerable magnitude such that it can not be neglected [Eckert and Drake (1972)]. Dursunkaya and Worek (1992) studied the diffusion-thermo and thermal-diffusion effects in transient and steady natural convection from a vertical surface. Recently, Anghel et al. (2000) included the Dufour and Soret effects on free convection boundary layer over a vertical surface embedded in a porous medium. Very recently, Postelnicu (2004) studied the influence of a magnetic field on heat and mass transfer by natural convection from vertical surfaces in porous media considering Soret and Dufour effects. Hence the objective of the present paper is to study the above-mentioned Dufour and Soret effects on steady free convection and mass transfer flow past a continuously moving semi-infinite vertical porous flat plate embedded in a porous medium under the influence of a transversely applied magnetic field.

\section{Mathematical Formulation:}

Let us consider the steady free convection and mass transfer flow of a viscous, incompressible and electrically conducting fluid past a continuously moving semi-infinite vertical porous plate embedded in a porous medium under the influence of a transversely applied magnetic field. The flow is assumed to be in the $x$-direction, which is taken along the plate in the upward direction and $y$-axis is normal to it. Initially it is assumed that the plate and the fluid are at the same temperature $T$ and the concentration level everywhere in the fluid is same. At time $t>0$, the plate temperature and concentration are instantly raised to $T_{w}\left(>T_{\infty}\right)$ and $C_{w}\left(>C_{\infty}\right)$, which are thereafter maintained constant, where $T_{\infty}$ and $C_{\infty}$ are the temperature and concentration respectively outside the boundary layer. The induced magnetic field is assumed to be negligible, such that $\boldsymbol{B}=\left(0, B_{0}, 0\right)$. The equation of conservation of electric charge $\nabla . \boldsymbol{J}=0$ gives $J_{y}=$ constant, where $\boldsymbol{J}=\left(J_{x}, J_{y}, J_{z}\right)$. Since the plate is electrically nonconducting, this constant is zero and hence $J_{y}=0$ everywhere in the flow. Assuming that the Boussinesq and boundary-layer approximations hold and using the Darcy-Forchheimer model, the governing equations relevant to the problem are given by:

Continuity equation

$$
\frac{\partial u}{\partial x}+\frac{\partial v}{\partial y}=0
$$

Momentum equation

$u \frac{\partial u}{\partial x}+v \frac{\partial u}{\partial y}=v \frac{\partial^{2} u}{\partial y^{2}}+g \beta\left(T-T_{\infty}\right)+g \beta^{*}\left(C-C_{\infty}\right)-\frac{\sigma B_{0}^{2} u}{\rho}-\frac{v u}{K}-\frac{b u^{2}}{K}$

Energy equation

$u \frac{\partial T}{\partial x}+v \frac{\partial T}{\partial y}=\alpha \frac{\partial^{2} T}{\partial y^{2}}+\frac{D_{m} k_{T}}{c_{s} c_{p}} \frac{\partial^{2} C}{\partial y^{2}}$

Concentration equation

$$
u \frac{\partial C}{\partial x}+v \frac{\partial C}{\partial y}=D_{m} \frac{\partial^{2} C}{\partial y^{2}}+\frac{D_{m} k_{T}}{T_{m}} \frac{\partial^{2} T}{\partial y^{2}},
$$


where the variables and related quantities are defined in the Nomenclature.

The boundary conditions for the model are given by:

$\left.\begin{array}{l}u=U_{0}, v=v_{0}(x), T=T_{w}, C=C_{w} \text { at } y=0, \\ u=0, v=0, T=T_{\infty}, C=C_{\infty} \text { as } y \rightarrow \infty,\end{array}\right\}$

where $U_{0}$ is the uniform velocity and $v_{0}(x)$ is the velocity of suction at the plate.

We now introduce the following dimensionless variables:

$$
\begin{aligned}
& \eta=y \sqrt{\frac{U_{0}}{2 v x}}, \\
& u=U_{0} f^{\prime}(\eta) \text {, } \\
& \theta(\eta)=\frac{T-T_{\infty}}{T_{w}-T_{\infty}}, \\
& \phi(\eta)=\frac{C-C_{\infty}}{C_{w}-C_{\infty}} .
\end{aligned}
$$

Also by introducing the relation (6) into equation (1) we obtain

$$
v=\sqrt{\frac{v U_{0}}{2 x}}\left(\eta f^{\prime}-f\right) \text {. }
$$

Introducing equations (6) and (7) into the equations (2)-(4) we obtain the following local similarity equations:

$$
\begin{aligned}
& f^{\prime \prime \prime}+f f^{\prime \prime}+G r \theta+G m \phi-M f^{\prime}-\frac{1}{D a \operatorname{Re}_{1}} f^{\prime}-\frac{F s_{1}}{D a} f^{\prime 2}=0, \\
& \theta^{\prime \prime}+\operatorname{Pr} f \theta^{\prime}+\operatorname{Pr} D u \phi^{\prime \prime}=0, \\
& \phi^{\prime \prime}+\operatorname{Sc} f \phi^{\prime}+\operatorname{ScS} \operatorname{Sr} \theta^{\prime \prime}=0,
\end{aligned}
$$

where $G r=\frac{g \beta\left(T_{w}-T_{\infty}\right) 2 x}{U_{0}^{2}}$ is the local Grashof number, $G m=\frac{g \beta^{*}\left(C_{w}-C_{\infty}\right) 2 x}{v U_{0}}$ is the local modified Grashof number, $M=\frac{\sigma B_{0}^{2} 2 x}{\rho U_{0}}$ is the Magnetic field parameter, $D a=\frac{K}{2 x^{2}}$ is the local Darcy number, $\operatorname{Re}_{1}=\frac{U_{0} x}{v}$ is the local Reynolds number, $F s_{1}=\frac{b}{x}$ is the local Forchheimer number, $\operatorname{Pr}=\frac{v}{\alpha}$ is the Prandtl number, $S c=\frac{v}{D_{m}}$ is the Schmidt number, $S r=\frac{D_{m} k_{T}\left(T_{w}-T_{\infty}\right)}{v T_{m}\left(C_{w}-C_{\infty}\right)}$ is the Soret number, $D u=\frac{D_{m} k_{T}\left(C_{w}-C_{\infty}\right)}{c_{s} c_{p} v\left(T_{w}-T_{\infty}\right)}$ is the Dufour number.

The boundary conditions are now transformed to:

$$
\left.\begin{array}{l}
f=f_{w}, f^{\prime}=1, \theta=1, \phi=1 \text { at } \eta=0, \\
f^{\prime}=0, \theta=0, \phi=0 \text { as } \eta \rightarrow \infty,
\end{array}\right\}
$$

where $f_{w}=-v_{0} \sqrt{\frac{2 x}{v U_{0}}}$ is the dimensionless suction velocity and prime denotes differentiation with respect to the variable $\eta$. 
The parameters of engineering interest for the present problem are the local skin-friction coefficient $\left(C_{f}\right)$, the local Nusselt number $(N u)$ and the local Sherwood number $(S h)$, which are given respectively by the following expressions:

$\frac{1}{2} \operatorname{Re}_{1}^{\frac{1}{2}} C_{f}=f^{\prime \prime}(0)$

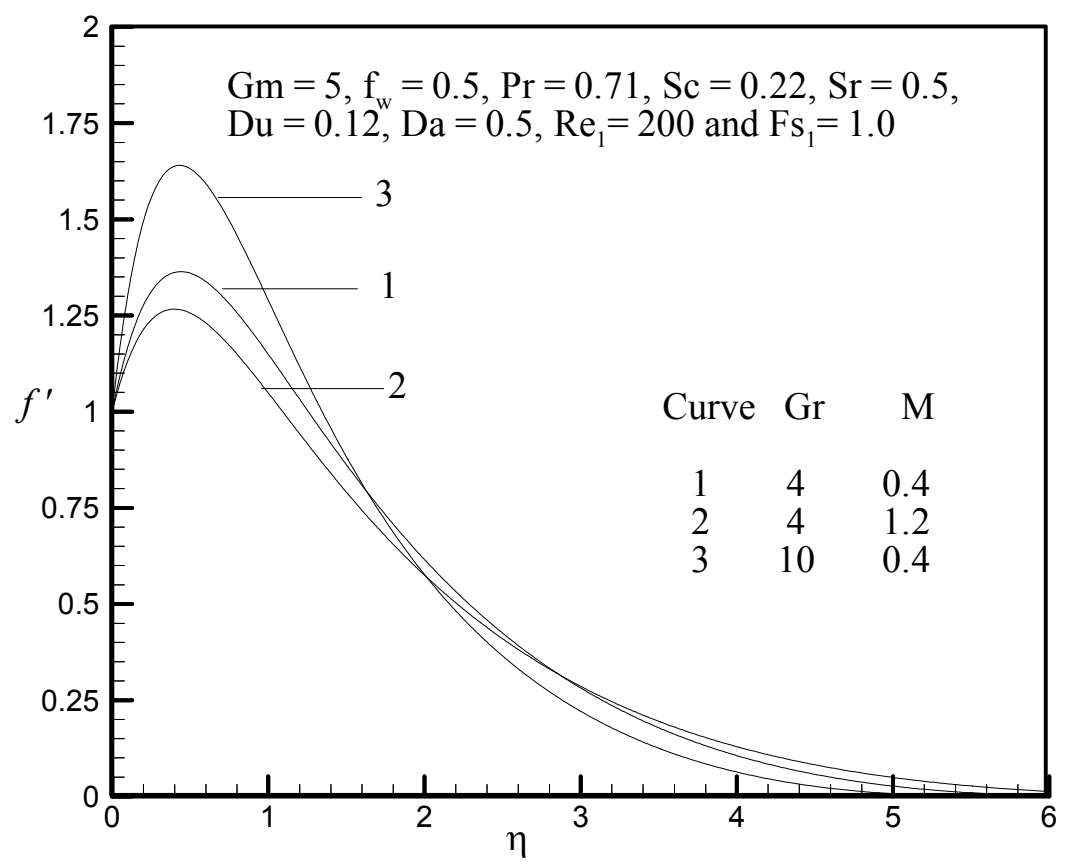

Fig. 1: Velocity profiles for different values of $\mathrm{Gr}$ and $\mathrm{M}$.

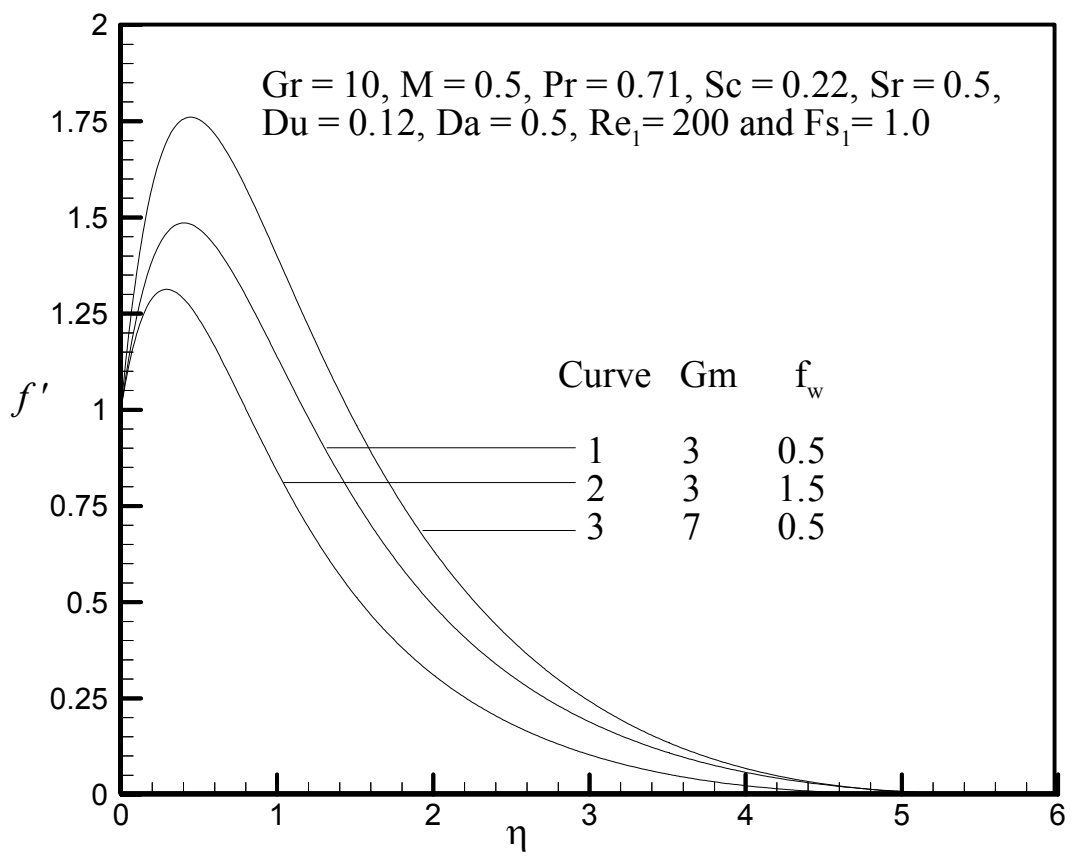

Fig. 2: Velocity profiles for different values of $\mathrm{Gm}$ and $\mathrm{f}_{\mathrm{w}}$. 
$N u\left(\operatorname{Re}_{1}\right)^{-\frac{1}{2}}=-\theta^{\prime}(0)$,
$S h\left(\operatorname{Re}_{1}\right)^{-\frac{1}{2}}=-\phi^{\prime}(0)$.

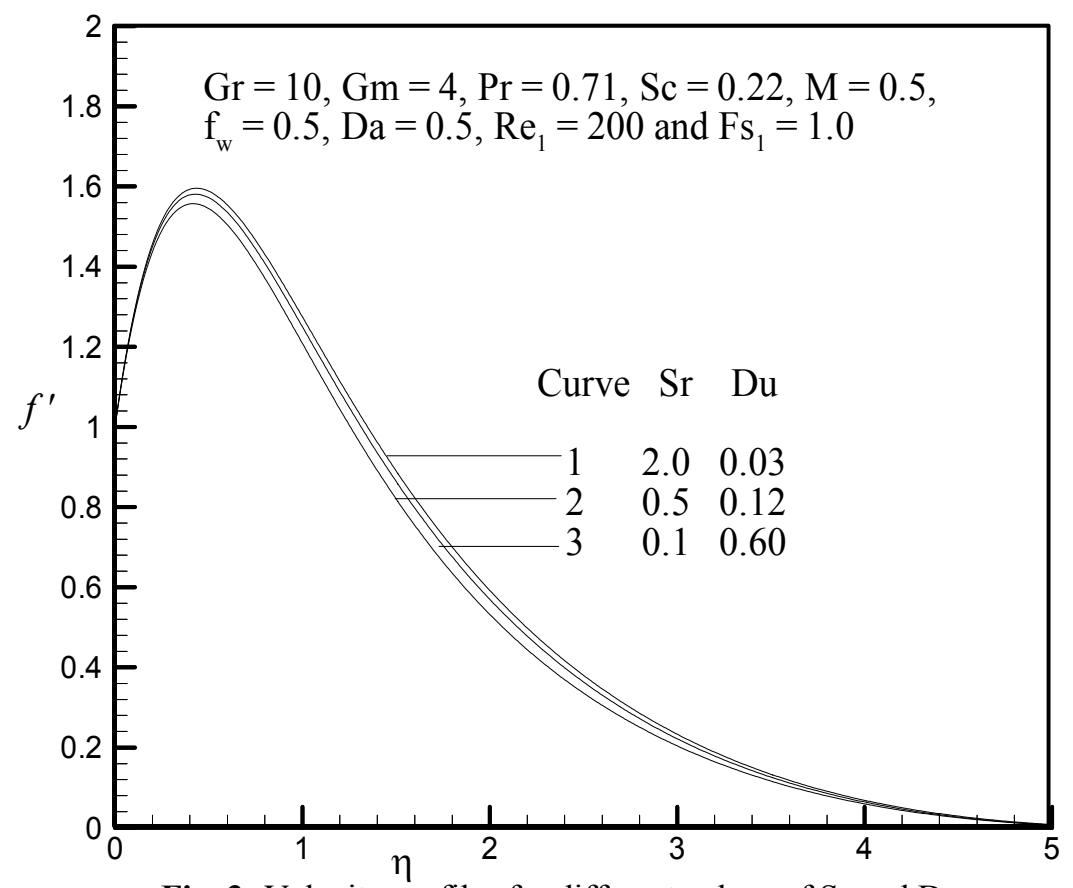

Fig. 3: Velocity profiles for different values of $\mathrm{Sr}$ and $\mathrm{Du}$.

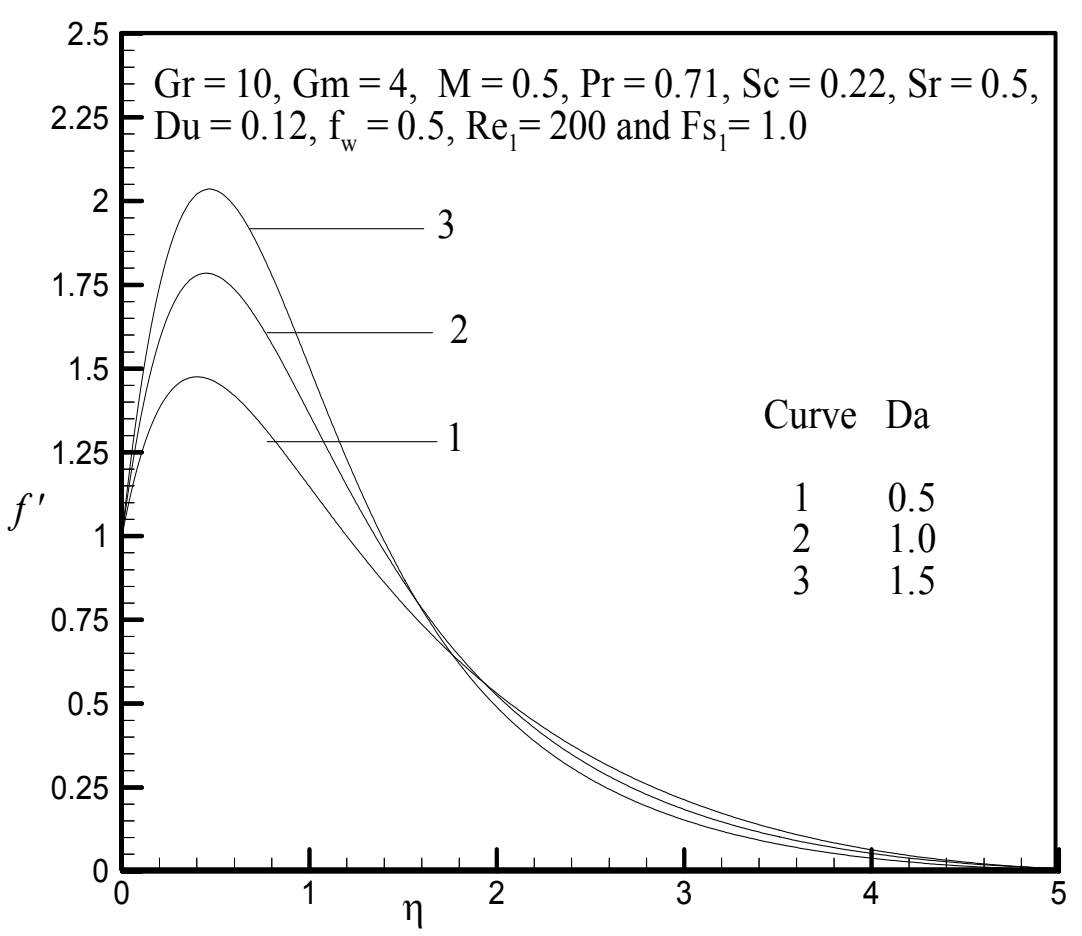

Fig. 4: Velocity profiles for different values of Da. 


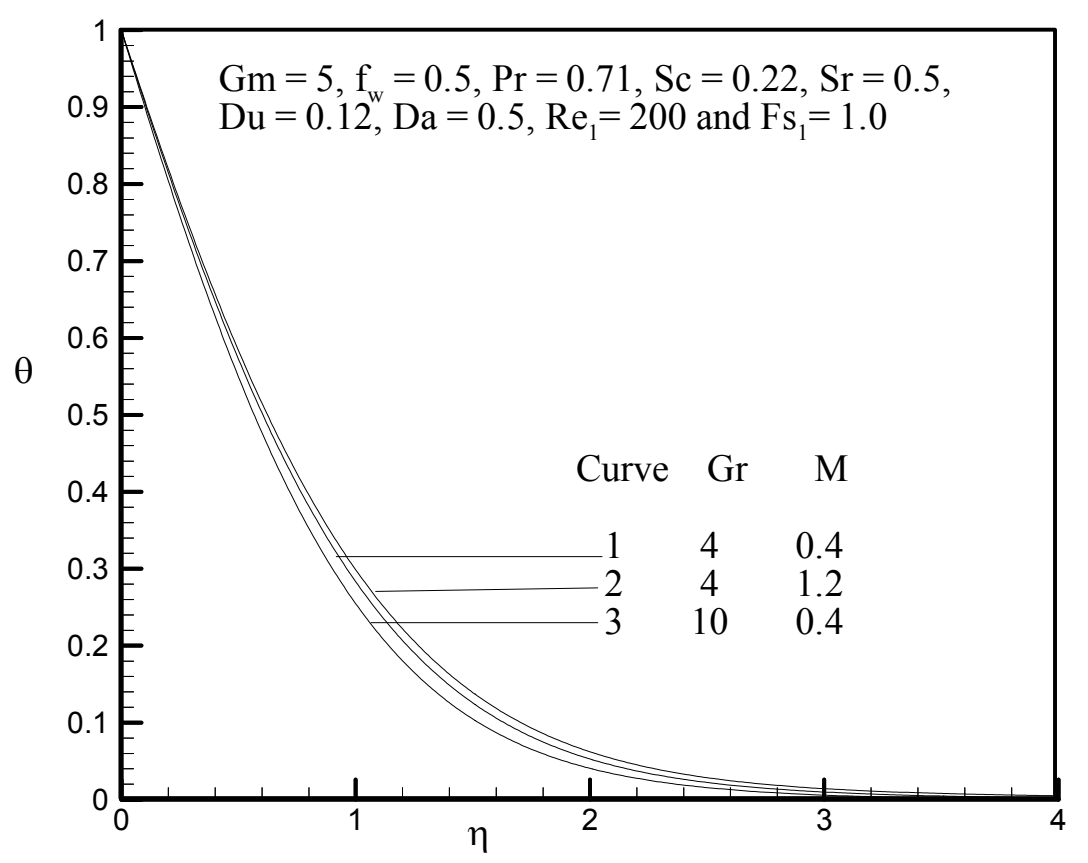

Fig. 5: Temperature profiles for different values of Gr and M.

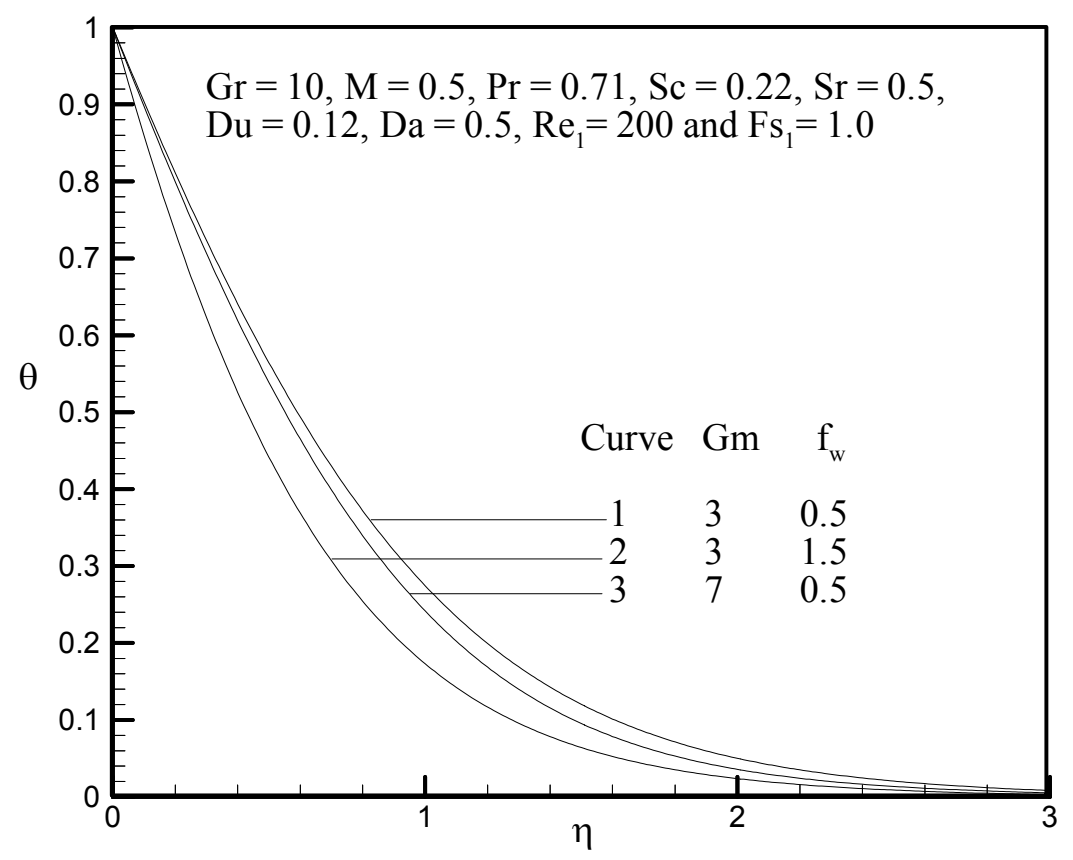

Fig. 6: Temperature profiles for different values of $\mathrm{Gm}$ and $\mathrm{f}_{\mathrm{w}}$.

The set of equations (8) - (10) under the boundary conditions (11) have been solved numerically by applying the Nachtsheim-Swigert (1965) shooting iteration technique together with Runge-Kutta sixthorder integration scheme. From the process of numerical computation, the skin-friction coefficient, the 
local Nusselt number and the local Sherwood number, which are respectively proportional to $f^{\prime \prime}(0)$, $-\theta^{\prime}(0)$ and $-\phi^{\prime}(0)$, are also worked out and their numerical values are presented in a tabular form.

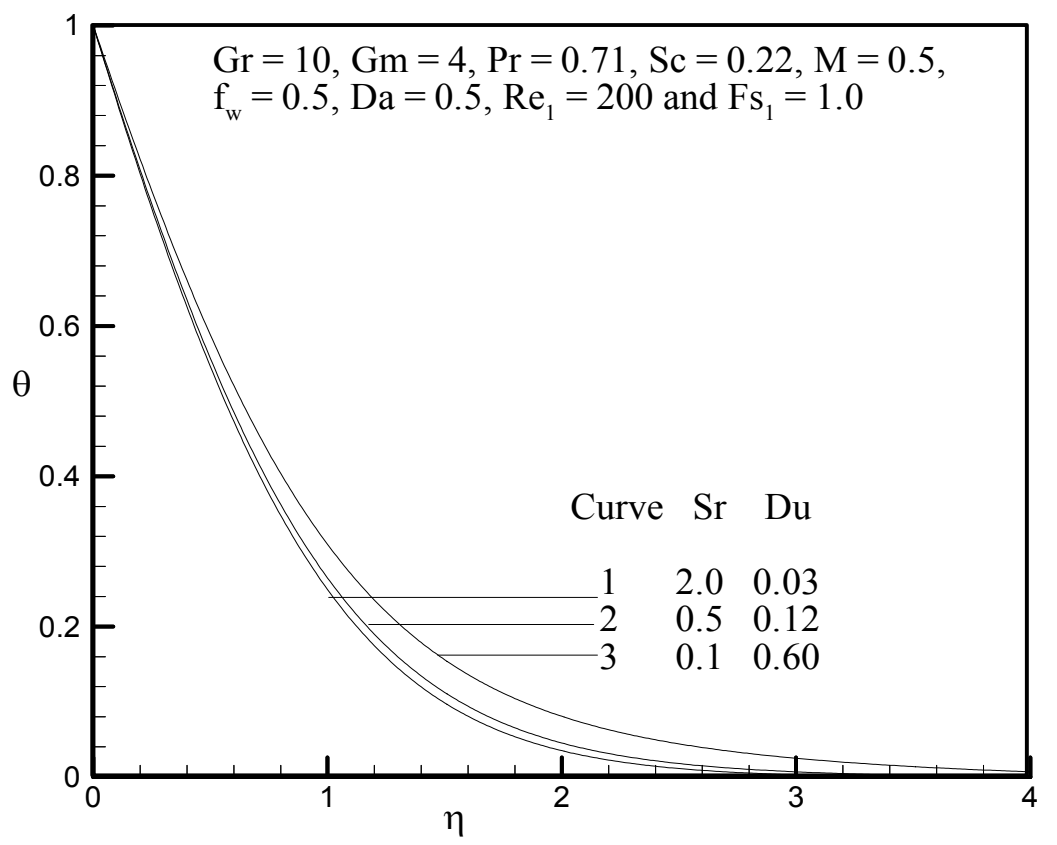

Fig. 7: Temperature profiles for different values of $\mathrm{Sr}$ and Du.

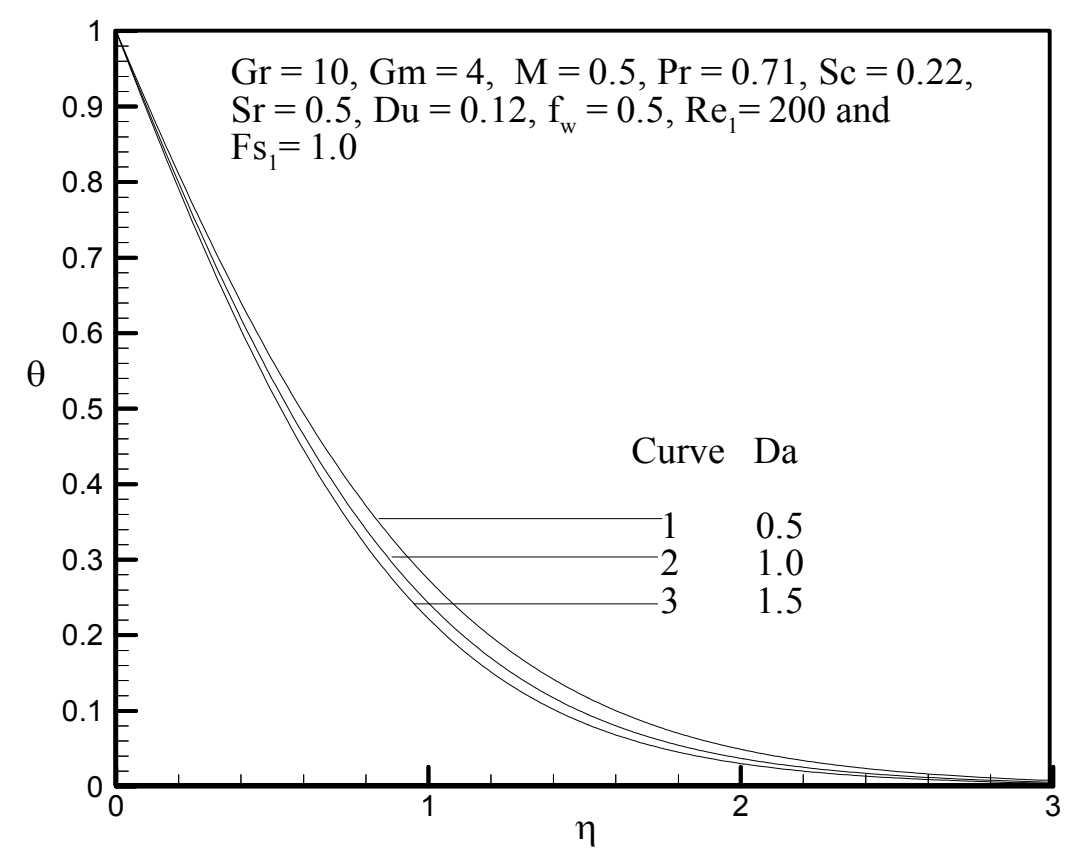

Fig. 8: Temperature profiles for different values of Da.

\section{Results and Discussion:}

During the course of the discussion of the effects of various parameters on the flow field the following considerations are made:

(i) The value of Prandtl number $\operatorname{Pr}$ is taken equal to 0.71 , which corresponds, physically to air. 
(ii) The value of Schmidt number $S c$ is chosen at 0.22 , which represents hydrogen at approx. $25^{\circ} \mathrm{C}$ and 1 atm.

(iii) The values of Dufour number $D u$ and Soret number $S r$ are chosen in such a way that their product is constant provided that the mean temperature $T_{m}$ is kept constant as well.

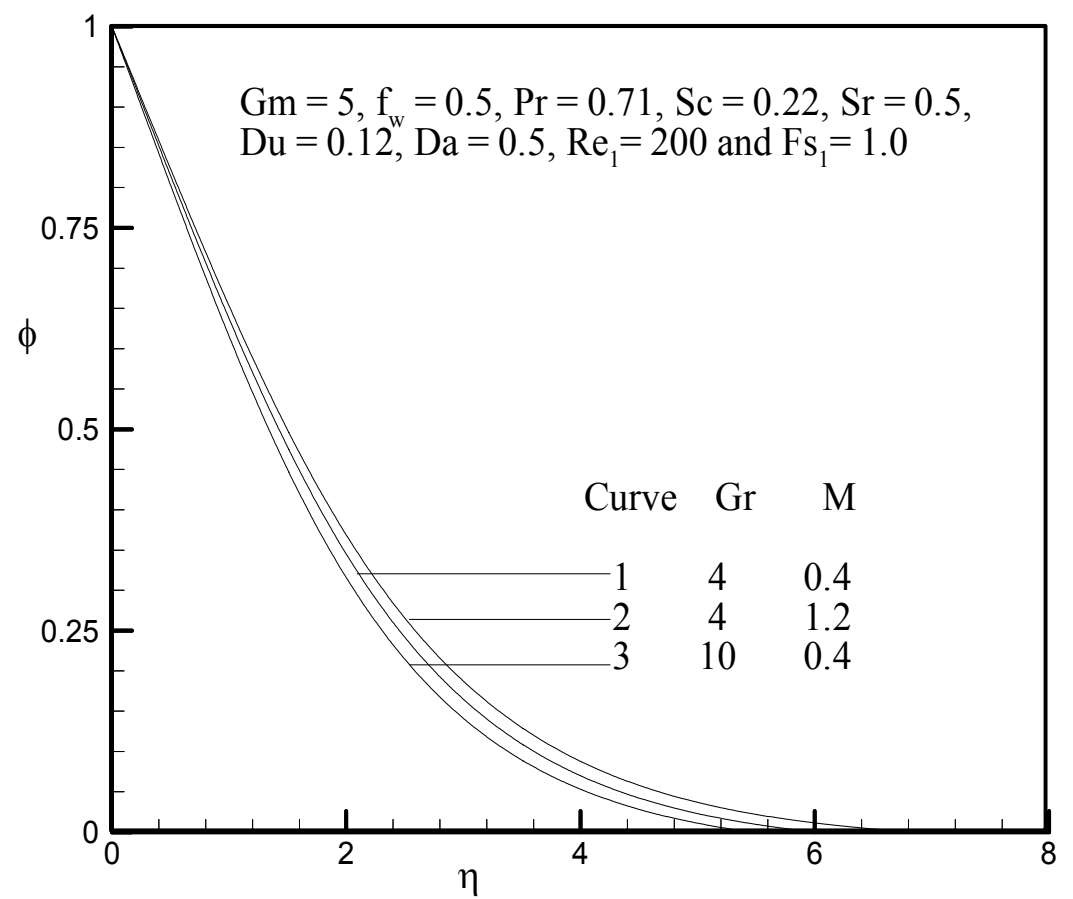

Fig. 9: Concentration profiles for different values of $\mathrm{Gr}$ and $\mathrm{M}$.

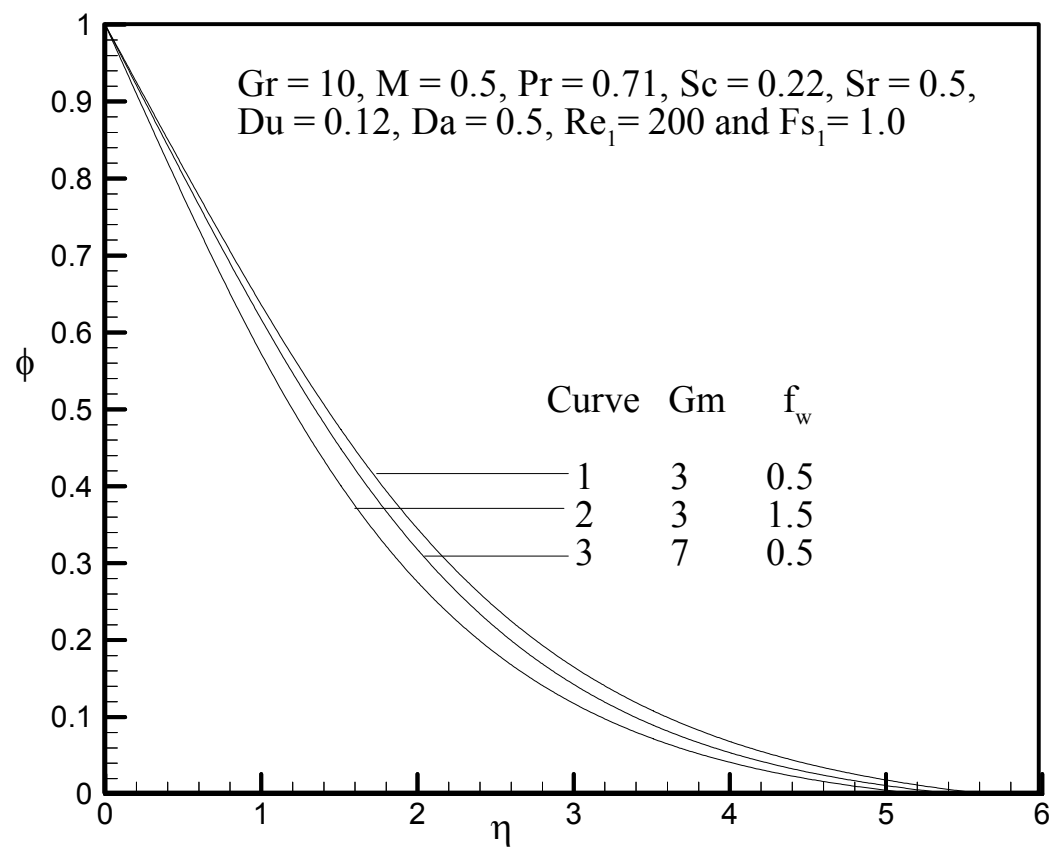

Fig. 10: Concemtration profiles for different values of $\mathrm{Gm}$ and $\mathrm{f}_{\mathrm{w}}$. 
(iv) Finally, the values of the local Grashof number Gr, local modified Grashof number Gm, suction parameter $f_{w}$, magnetic field parameter $M$, local Reynolds number $R e_{l}$, local Darcy number $D a$ and local Forchheimer number $F s_{1}$ are chosen arbitrarily.

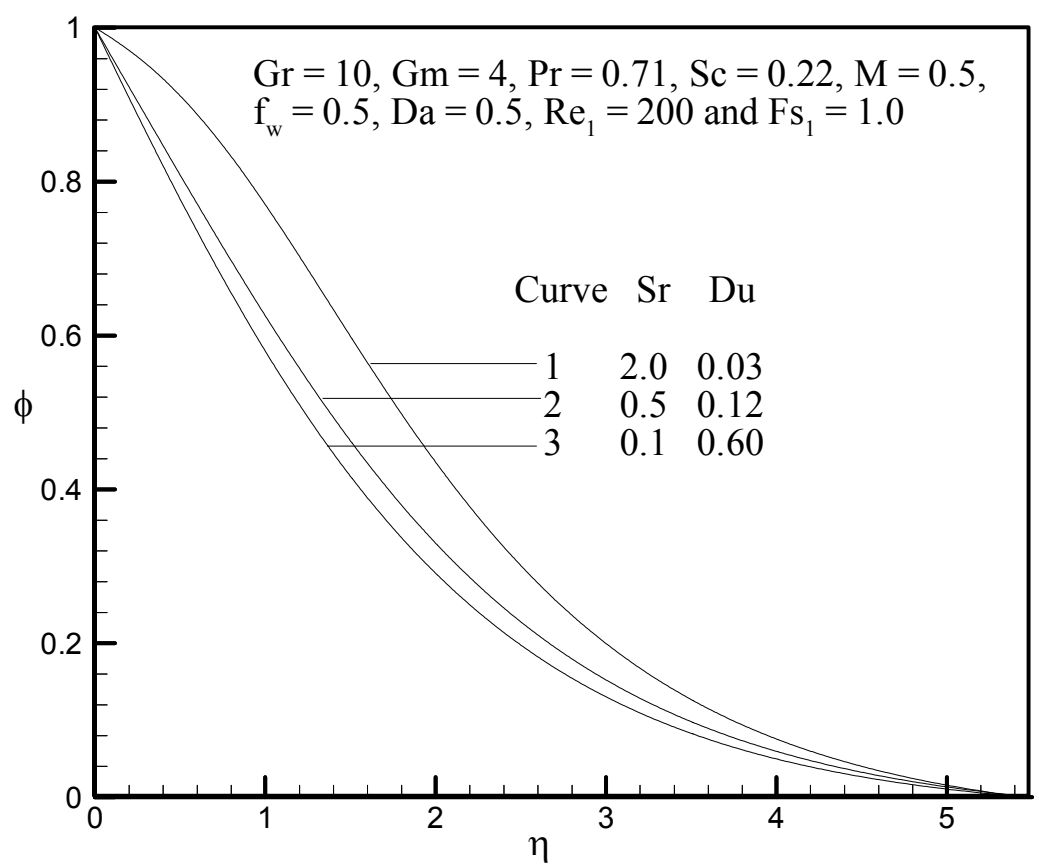

Fig. 11: Concentration profiles for different values of $\mathrm{Sr}$ and $\mathrm{Du}$.

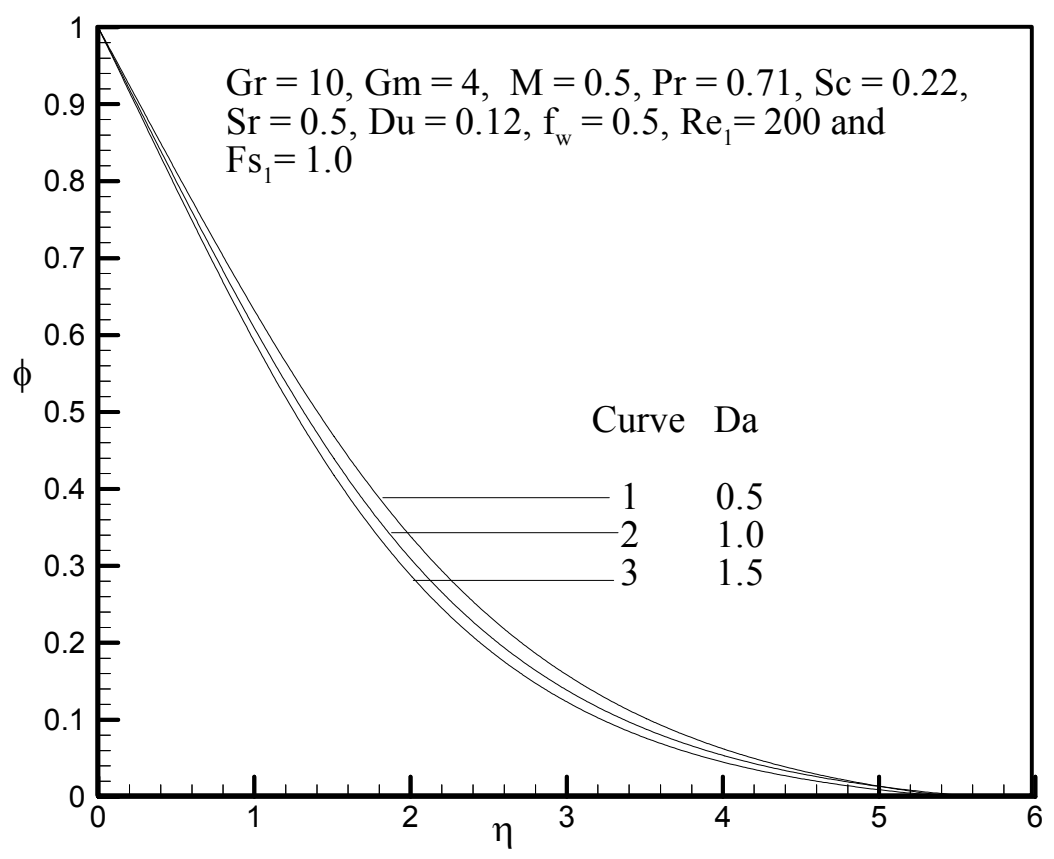

Fig. 12: Concentration profiles for different values of Da. 
Under the above assumptions, results are shown in Figs. 1-12 and in Table 1. The effects of Grashof number and magnetic field parameter on the velocity field are shown in Fig.1. It is seen from this figure that the velocity decreases with the increase of magnetic field parameter while it increases with the increase of Grashof number (or increase of free convection current). In Fig.2 the effects of modified Grashof number and suction parameter on the velocity field are shown. Fig. 2 shows that the velocity increases when the concentration difference between the mean and free stream values increases, whereas it decreases with an increase of suction parameter indicating suction stabilizes the boundary layer growth.

The influence of Soret number $S r$ and Dufour number $D u$ on the velocity field are shown in Fig. 3. Quantitatively, when $\eta=1.0$ and $\mathrm{Sr}$ decreases from 2 to 0.5 (or $D u$ increases from 0.03 to 0.12 ), there is $5.48 \%$ decrease in the velocity. On the other hand, when $S r$ decreases from 0.5 to 0.1 , there is $3.42 \%$ increase in the velocity. The effect of Darcy number $D a$ on the velocity field is shown in Fig. 4. From this figure we observe that velocity increases with the increase of Darcy number. For large Darcy number porosity of the medium increases, hence fluid flows quickly.

The effects of Grashof number and magnetic field parameter on the temperature field are shown in Fig. 5. From this figure we observe that the temperature increases with an increase of magnetic field parameter and decreases with an increase of free convection current. The effects of modified Grashof number and suction parameter on the temperature profiles are shown in Fig.6. This figure shows that the temperature decreases with the increase of both suction parameter and modified Grashof number. From Fig. 7 when $\eta=1.0$ and $S r$ decreases from 2 to 0.5 (or $D u$ increases from 0.03 to 0.12 ), there is $6.19 \%$ increase in the temperature, whereas the corresponding increase is $16.97 \%$ when $\mathrm{Sr}$ decreases from 0.5 to 0.1 . The effect of Darcy number $D a$ on the temperature field is shown in Fig. 8. From this figure we observe that temperature decreases with the increase of Darcy number. As the fluid velocity increases with the increase of the Darcy number, consequently temperature surrounding the plate decreases.

Table 1: Numerical values of skin-friction coefficient $\left(C_{f}\right)$, Nusselt number $(N u)$ and Sherwood number $(S h)$ for $\mathrm{Gr}=10, \mathrm{Gm}=4, \mathrm{Pr}=0.71, \mathrm{Sc}=0.22, \mathrm{f}_{\mathrm{w}}=0.5, \mathrm{M}=0.5, \mathrm{Da}=0.5, \mathrm{Re}_{1}=200$ and $\mathrm{Fs}_{1}=1.0$

\begin{tabular}{|c|c|c|c|c|}
\hline Sr & $\boldsymbol{D u}$ & $\boldsymbol{C}_{\boldsymbol{f}}$ & $\boldsymbol{N u}$ & $\boldsymbol{S h}$ \\
\hline 2.0 & 0.03 & 3.4231141 & 1.0283189 & 0.1296854 \\
\hline 1.0 & 0.06 & 3.3457474 & 1.0155338 & 0.2992750 \\
\hline 0.5 & 0.12 & 3.3162482 & 1.0019868 & 0.3844602 \\
\hline 0.4 & 0.15 & 3.3141130 & 0.9965224 & 0.4017999 \\
\hline 0.2 & 0.30 & 3.3287043 & 0.9718535 & 0.4381199 \\
\hline 0.1 & 0.60 & 3.3828661 & 0.9248360 & 0.4602605 \\
\hline
\end{tabular}

The effects of Grashof number and magnetic field parameter on the concentration field are shown in Fig. 9. From this figure we observe that the concentration increases with an increase of magnetic field parameter and decreases with an increase of free convection current. The effects of modified Grashof number and suction parameter on the concentration profiles are shown in Fig. 10. This figure shows that the concentration decreases with the increase of suction parameter as well as the modified Grashof number. In Fig. 11 when $\eta=1.0$ and $S r$ decreases from 2 to 0.5 (or $D u$ increases from 0.03 to 0.12 ), there is $23.11 \%$ decrease in the concentration, whereas the corresponding decrease is $7.91 \%$ when $\mathrm{Sr}$ decreases from 0.5 to 0.1 . The effect of Darcy number $D a$ on the concentration field is shown in Fig. 12. This figure reveals that concentration of the fluid within the boundary layer decreases with the increase of Darcy number. 
Finally, the effects of Soret and Dufour numbers on the skin-friction coefficient, Nusselt number and Sherwood number are shown in Table 1. The behaviour of these parameters is self-evident from the Table 1 and hence they will not discuss any further due to brevity.

\section{Conclusions}

In this paper we have studied numerically the Dufour and Soret effects on a steady MHD free convention and mass transfer flow past a semi-infinite vertical plate embedded in a porous medium. From the present study the following conclusions can be drawn:

- The velocity profiles decrease whereas temperature and concentration profiles increase with an increase of magnetic field parameter.

- The velocity profiles increase whereas temperature and concentration profiles decrease with an increase of free convection currents.

- The suction stabilizes the boundary layer growth.

- Large Darcy number (large porosity of the medium) leads to the increase of the velocity and decrease of the temperature as well as concentration of the fluid within the boundary layer.

- For fluids with medium molecular weight $\left(\mathrm{H}_{2}\right.$, air), Dufour and Soret effects should not be neglected.

\section{References}

Anghel, M., Takhar, H. S. and Pop, I. (2000): Dufour and Soret effects on free-convection boundary layer over a vertical surface embedded in a porous medium, Studia Universitatis Babes-Bolyai, Mathematica Vol. XLV, pp. 11-21.

Cramer, K. R. and Pai, S. I. (1973): Magnetofluid dynamics for Engineers and applied Physicists, McGraw-Hill Co., New York.

Dursunkaya. Z. and Worek, W. M. (1992): Diffusion-thermo and thermal-diffusion effects in transient and steady natural convection from vertical surface, Int. J. Heat Mass Transfer, Vol. 35, pp. 20602065.

Eckert, E. R. G. and Drake, R. M. (1972): Analysis of Heat and Mass Transfer, McGraw-Hill, New York.

Gupta, A. S. (1961): Steady and transient free convection of an electrically conducting fluid from a vertical plate in the presence of magnetic field, Appl. Sci. Res., Vol. 9A, pp. 319-333.

Lykoudis, P. S. (1962): Natural convection of an electrically conducting fluid in the presence of a magnetic field, Int. J. Heat Mass Transfer, Vol. 5, pp. 23-34.

Nanda, R. S. and Mohanty, H. K. (1970): Hydromagnetic free convection for high and low Prandtl numbers, J. Phys. Soc. Japan, Vol. 29, pp. 1608-1618.

Nachtsheim, P. R. and Swigert, P. (1965): Satisfaction of the asymptotic boundary conditions in numerical solution of the system of nonlinear equations of boundary layer type, NASA TND-3004.

Postelnicu, A. (2004): Influence of a magnetic field on heat and mass transfer by natural convection from vertical surfaces in porous media considering Soret and Dufour effects, Int. J. Heat Mass Transfer, Vol. 47, pp. 1467-1472.

Raptis, A. and Kafoussias, N. G. (1982): Magnetohydrodynamic free convection flow and mass transfer through porous medium bounded by an infinite vertical porous plate with constant heat flux, Can. J. Phys. Vol. 60, pp. 1725-1729.

Sattar, M. A., Rahman, M. M. and Samad, M. A. (2001): Similar solutions of an MHD free convection and mass transfer flow with viscous dissipation, Dhaka Univ. J. Sci., Vol. 49, pp. 67-78. 